\title{
Highly Sensitive CO Gas Sensor from Defective Graphene: Role of van der Waals Interactions
}

\author{
Yingda Jiang, Sha Yang, Shuang Li, Wei Liu, and Yonghao Zhao \\ Nano Structural Materials Center, School of Materials Science and Engineering, Nanjing University of Science and Technology, \\ Nanjing, Jiangsu 210094, China \\ Correspondence should be addressed to Wei Liu; weiliu@njust.edu.cn
}

Received 20 May 2015; Accepted 11 June 2015

Academic Editor: Xuping Sun

Copyright (C) 2015 Yingda Jiang et al. This is an open access article distributed under the Creative Commons Attribution License, which permits unrestricted use, distribution, and reproduction in any medium, provided the original work is properly cited.

\begin{abstract}
Layered materials, such as graphene, have attracted increasing interests since they can be extensively used in gas sensors, spintronic devices, and transparent electrodes. Although larger size of graphene sheets has been fabricated, in reality, the existence of the defects in layered materials is almost inevitable during the manufacturing process. Here, we performed the state-of-the-art densityfunctional theory calculations to study the interactions between $\mathrm{CO}$ molecule and the pristine and defective graphene layers, with the aim of designing a CO gas sensor with higher sensitivity. The van der Waals interactions predominate the binding between the $\mathrm{CO}$ gas and the sensor, and also significantly enhance the stability of the system. The defective graphene strongly interacts with $\mathrm{CO}$, and thus enhances the sensitivity of the graphene and further tunes the electronic and magnetic properties of the entire system. Our computed results clearly demonstrate that the defective graphene could be a good sensor for gas molecules.
\end{abstract}

\section{Introduction}

Carbon-based materials, such as nanotubes and graphene, are widely accepted as a good sensor to monitor small gas molecules, such as $\mathrm{CO}, \mathrm{NO}, \mathrm{NO}_{2}$, and $\mathrm{NH}_{3}$ molecules [19]. In particular, the detection of the $\mathrm{CO}$ molecule to prevent poisoning is of vital importance, due to the fact that $\mathrm{CO}$ is colorless, tasteless, and odorless, but toxic and flammable. Carbon nanotubes were often used as building blocks in gas sensors, which is capable of effectively monitoring extremely low concentrations of gases [10-13]. Since fabrication in 2004 by Novoselov et al. [14], graphene, the two-dimensional material, has attracted increasing interests as a gas sensor, thanks to its huge specific surface area, good electrical conductivity, stable chemical performance, high Young's modulus, and excellent optical performance $[3,8,15-17]$. In particular, Zhang et al. [16] theoretically studied small gas molecules (CO, $\mathrm{NO}, \mathrm{NO}_{2}, \mathrm{NH}_{3}$ ) adsorption on four graphene layers (pristine, $\mathrm{B}$ and $\mathrm{N}$-doped, and defective graphene) using the local-density approximation (LDA) method and exploited their potential applications as gas sensors. Although efforts are being undertaken to enhance the performance of gas sensor, many challenges remain in the field, such as the low binding energy, low sensitivity, and bad selectivity.

A prerequisite to design an effective sensor is the understanding of the interactions between the gas molecules and the substrate. However, from the point of view of modelling, the accurate description of their interactions remains a big challenge, due to the well-known fact that the gas/substrate binding arises from the dedicated balance between various types of bonding: covalent bonding, van der Waals (vdW) interactions, hydrogen bonding, charge transfer, and Pauli repulsion $[15,18-20]$. Thanks to the recent development of $\mathrm{vdW}$-inclusive methods in the framework of densityfunctional theory (DFT) [21, 22], the role of $\mathrm{vdW}$ force between small molecules in the gas phase has been reasonably described and is well understood. Notably, the vdW energy is of extreme importance in two-dimensional materials but is often ignored in previous DFT calculations when studying the gas sensors.

In this contribution, we performed various types of vdWinclusive DFT calculations to study the interactions between $\mathrm{CO}$ molecule and graphene layers. Although larger size of graphene sheets has been fabricated, in reality, the existence 
TABLE 1: Adsorption energy ( $E_{\mathrm{ad}}$ in $\left.\mathrm{eV}\right)$ and equilibrium molecule-substrate distance $(d$ in $\AA$ ) of the CO adsorbed on different sites.

\begin{tabular}{|c|c|c|c|c|c|c|c|}
\hline \multirow{2}{*}{ Systems } & \multirow{2}{*}{ Structures } & \multicolumn{2}{|c|}{ LDA } & \multicolumn{2}{|c|}{ PBE } & \multicolumn{2}{|c|}{$\mathrm{PBE}+\mathrm{vdW}(\mathrm{TS})$} \\
\hline & & $E_{\mathrm{ad}}$ & $d$ & $E_{\text {ad }}$ & $d$ & $E_{\text {ad }}$ & $d$ \\
\hline \multirow{10}{*}{$\mathrm{CO}$ on $\mathrm{G}$} & $\mathrm{a}$ & -0.10 & 3.01 & 0.03 & 3.14 & -0.05 & 2.84 \\
\hline & $\mathrm{b}$ & -0.04 & $2.72^{\mathrm{a}}$ & 0.04 & $2.97^{\mathrm{a}}$ & -0.03 & $2.68^{\mathrm{a}}$ \\
\hline & c & -0.08 & 2.82 & 0.02 & 3.22 & -0.03 & 2.77 \\
\hline & $\mathrm{d}$ & -0.03 & $2.71^{\mathrm{a}}$ & 0.05 & $2.85^{\mathrm{a}}$ & -0.04 & $2.71^{\mathrm{a}}$ \\
\hline & e & -0.08 & 2.61 & 0.06 & 2.85 & -0.03 & 2.76 \\
\hline & $\mathrm{f}$ & -0.06 & $2.63^{\mathrm{a}}$ & 0.07 & $2.72^{\mathrm{a}}$ & 0.03 & $2.47^{\mathrm{a}}$ \\
\hline & g & -0.11 & 2.86 & 0.04 & 3.10 & -0.10 & 2.79 \\
\hline & $\mathrm{h}$ & -0.12 & 2.69 & 0.04 & 2.97 & -0.11 & 2.88 \\
\hline & $\mathrm{i}$ & -0.12 & 2.83 & 0.03 & 3.09 & -0.09 & 2.85 \\
\hline & j & -0.12 & 2.82 & 0.03 & 3.14 & -0.10 & 2.91 \\
\hline \multirow{2}{*}{ CO on defective $\mathrm{G}$} & $\mathrm{k}$ & -0.36 & 2.90 & -0.25 & 3.09 & -0.32 & 2.63 \\
\hline & 1 & -2.75 & $1.32^{\mathrm{b}}$ & -2.05 & $1.33^{\mathrm{b}}$ & -2.15 & $1.33^{\mathrm{b}}$ \\
\hline
\end{tabular}

a refers to the distance between the $\mathrm{O}$ atom in $\mathrm{CO}$ and the $\mathrm{C}$ atom in graphene.

$\mathrm{b}$ refers to the shortest bond length.

of the defects in layered materials is almost inevitable during the manufacturing process. As such it is of fundamental interests to study the interactions of gas molecules with the defective graphene sheets. Our calculations showed that by introducing defects, even a single point vacancy, in the graphene layers, the sensitivity of the gas sensor can be significantly enhanced. Moreover, the electric and magnetic properties of the system are strongly perturbed upon interaction with the molecules. We demonstrate that the defective graphene would be a highly sensitive sensor for monitoring the CO gas.

\section{Methods}

DFT calculations were performed using the Vienna ab initio simulation package (VASP) [23]. The interaction between the valence electrons and ionic cores is described by the projector augmented wave (PAW) method [24]. The LDA, the Perdew-Burke-Ernzerhof (PBE) form of the generalizedgradient approximation (GGA) [25], and the PBE + vdW (or TS) [26] are employed to describe electron exchange and correlation. The plane wave energy cut-off is set to $350 \mathrm{eV}$ and the Brillouin zone is sampled with $3 \times 3 \times 1 k$-point mesh. The surfaces are modeled by periodic $(5 \times 5)$ supercell, which is separated by $15 \AA$ of vacuum. A threshold for the convergence criteria of $0.001 \mathrm{eV} / \AA$ is used for the final force. For the calculation of density of electronic states (DOS), the $k$-point is set to $19 \times 19 \times 1$.

The adsorption energy of CO on graphene can be written as

$$
E_{\mathrm{ads}}=E_{\mathrm{CO} / \text { Graphene }}-E_{\mathrm{Graphene}}-E_{\mathrm{CO}},
$$

where $E_{\mathrm{CO} / \text { Graphene }}, E_{\text {Graphene }}$, and $E_{\mathrm{CO}}$ are the total energies of the relaxed $\mathrm{CO}$ on graphene system, free-standing graphene, and the isolated CO molecule, respectively.

To find the most favorable adsorption configuration, a CO molecule is initially placed at different positions above the pristine graphene $(G)$ and the defective graphene (DG) with perpendicular or parallel orientation. We also use other five vdW-inclusive methods, including DFT-D2 [27], DFTD3 [28], vdW-DF, optPBE-vdW, and optB88-vdW [29, 30], to revisit the favorable adsorption configurations relaxed from the PBE + vdW method, with the aim of deeply understanding the role of vdW interactions in these adsorption systems.

\section{Results and Discussion}

Figure 1 shows the optimized configurations after full relaxation. The $\mathrm{C}-\mathrm{C}$ bond length in graphene from our LDA, $\mathrm{PBE}$, and $\mathrm{PBE}+\mathrm{vdW}$ calculations ranges from 1.41 to $1.43 \AA$, in excellent agreement with the experimental data $(1.42 \AA)$ [31]. Also, the calculated atomic distance between $\mathrm{O}$ and $\mathrm{C}$ in $\mathrm{CO}$ molecule agrees very well with those from experiments (1.13 to $1.14 \AA$ versus $1.13 \AA$ [32]). The computed adsorption energies $\left(E_{\mathrm{ad}}\right)$ and the equilibrium moleculesubstrate distances $(d)$ are listed in Table 1 . The most stable configurations of the $\mathrm{CO}$ molecule on pristine graphene and defective graphene are configurations (h) and (l), respectively. The adsorption energy of CO on $\mathrm{G}$ is found to be $-0.12 \mathrm{eV}$, along with the CO-substrate distance of $2.69 \AA$ from the LDA functional. We found that the $E_{\text {ad }}$ of configurations (g) to $(\mathrm{j})$ are almost identical, suggesting the flat potentialenergy surface for $\mathrm{CO}$ at the pristine graphene. Notably, our computed adsorption energy at the favorable configuration (h) agrees with that in [16]. The LDA results are close to those from the PBE + vdW method with an $E_{\mathrm{ad}}$ of $-0.11 \mathrm{eV}$. In contrast, the PBE adsorption energies are positive, and the adsorption distances are considerably larger than those from $\mathrm{LDA}$ and $\mathrm{PBE}+\mathrm{vdW}$. Therefore, one could conclude that the GGA-PBE method tends to underestimate the adsorption energy and overestimate the adsorption distance, confirming the essential role of vdW interactions in these systems.

For the defective graphene, the configuration (l) in Figure 1 is found to be the most stable, where the CO tilts to the defective graphene and carbon atom pointing towards the vacancy. When using LDA, the adsorption energy of $\mathrm{CO}$ on DG significantly increases to $-2.75 \mathrm{eV}$, with a COsubstrate distance of $1.32 \AA$. The $E_{\text {ad }}$ computed from PBE and 


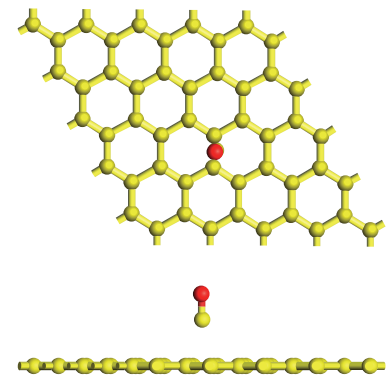

(a)

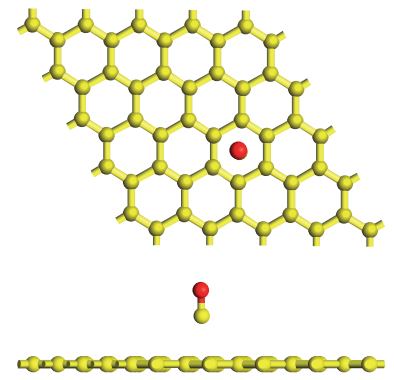

(e)

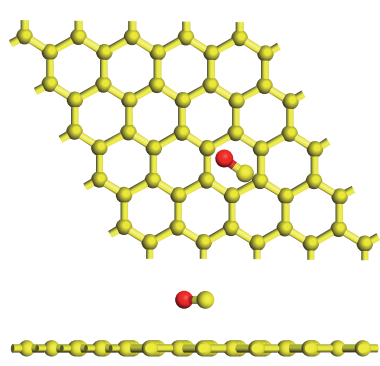

(i)

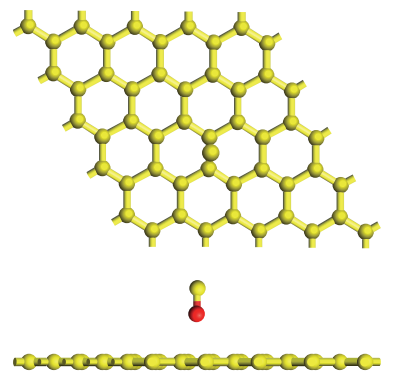

(b)

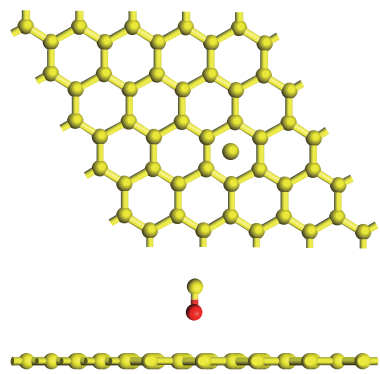

(f)

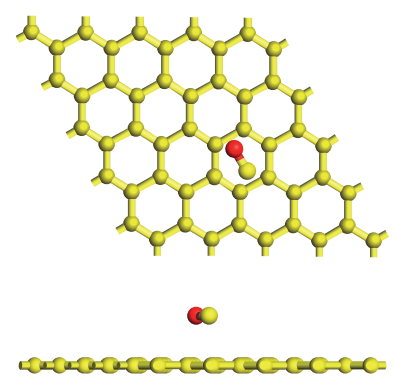

(j)

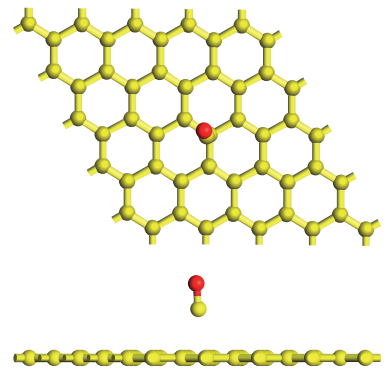

(c)

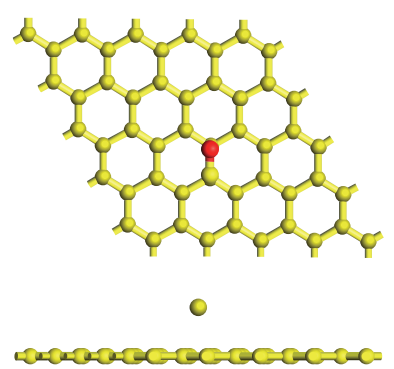

(g)

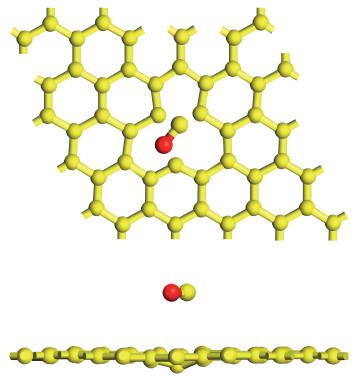

(k)

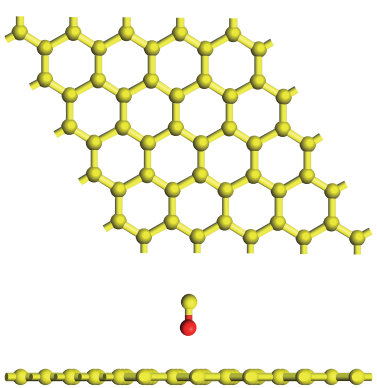

(d)

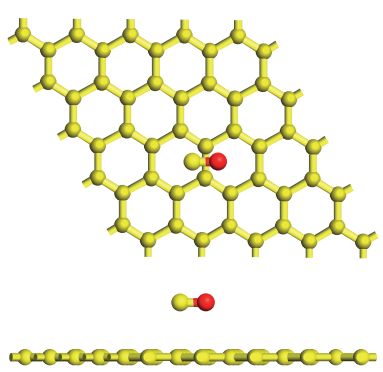

(h)

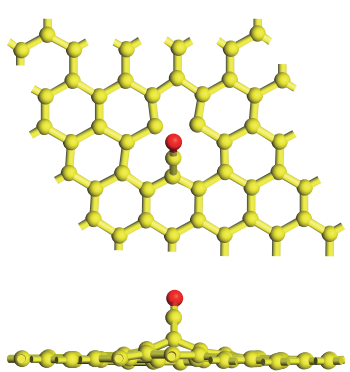

(l)

FIgURE 1: Top and side views of the adsorption structures for $\mathrm{CO}$ on graphene and defective graphene. (a) to ( $\mathrm{j}$ ) are for graphene and (k) to (l) are for defective graphene.

$\mathrm{PBE}+\mathrm{vdW}$ are -2.05 and $-2.15 \mathrm{eV}$, respectively, and the $d$ value dramatically decreases to $1.33 \AA$. The distance is close to the bond length of a $\mathrm{C}=\mathrm{C}$ double bond $(1.34 \AA)$, indicating the formation of the covalent bonds upon $\mathrm{CO}$ adsorption. As such, the CO molecule is chemisorbed at the defective graphene, with a significantly larger adsorption energy than for the pristine graphene sheet.

Table 2 shows the comparison of calculated adsorption energy and equilibrium molecule-substrate distance at the most stable sites $h$ and $l$ of $G$ and DG, respectively, between six $\mathrm{vdW}$ functionals. For configuration $\mathrm{h}$, the $E_{\mathrm{ad}}$ from DFT-D2 $(-0.09 \mathrm{eV})$ and DFT-D3 $(-0.10 \mathrm{eV})$ are lower than that of PBE $+\mathrm{vdW}(-0.11 \mathrm{eV})$, and the vdW-DF $(-0.14 \mathrm{eV})$ and optPBE$\mathrm{vdW}(-0.16 \mathrm{eV})$ are higher. The best agreement can be found in the optB88-vdW functional, with an $E_{\mathrm{ad}}=-0.11 \mathrm{eV}$. A relatively wide range of $E_{\text {ad }}(-0.09$ to $-0.16 \mathrm{eV})$ can be seen for the vdW-DF-type functionals, while the distance is from 2.77 to $3.02 \AA$. For configuration 1 , the $E_{\mathrm{ad}}$ values of DFT-D2 $(-2.25 \mathrm{eV})$ and DFT-D3 $(-2.17 \mathrm{eV})$ are higher than that of PBE $+\mathrm{vdW}(-2.15 \mathrm{eV})$, the vdW-DF $(-1.80 \mathrm{eV})$, optB88-vdW (-2.04 eV), and optPBE-vdW (-2.01 eV) which
TABLE 2: Comparison of the calculated adsorption energy $\left(E_{\mathrm{ad}}\right.$ in $\mathrm{eV})$ and equilibrium molecule-substrate distance ( $d$ in $\AA$ ) at sites $\mathrm{h}$ and $\mathrm{l}$ of graphene and defective graphene, respectively, between different vdW functionals.

\begin{tabular}{lcccc}
\hline Methods & \multicolumn{2}{c}{$\mathrm{h}$} & \multicolumn{2}{c}{$\mathrm{l}$} \\
& $E_{\mathrm{ad}}$ & $d$ & $E_{\mathrm{ad}}$ & $d$ \\
\hline PBE + vdW & -0.11 & 2.88 & -2.15 & 1.33 \\
DFT-D2 & -0.09 & 2.87 & -2.25 & 1.33 \\
DFT-D3 & -0.10 & 2.92 & -2.17 & 1.33 \\
vdW-DF & -0.14 & 3.02 & -1.80 & 1.33 \\
optB88-vdW & -0.11 & 2.77 & -2.04 & 1.33 \\
optPBE-vdW & -0.16 & 2.93 & -2.01 & 1.33 \\
\hline
\end{tabular}

are lower. Further analysis of the data in Table 2 shows that different vdW-inclusive methods give a relatively wide range in adsorption energies for the chemisorbed system. More specifically, the PBE + vdW and DFT-D3 give almost identical binding energies, which are about $100 \mathrm{meV}$ larger than those from the DFT-D2 method. This is presumably because the 


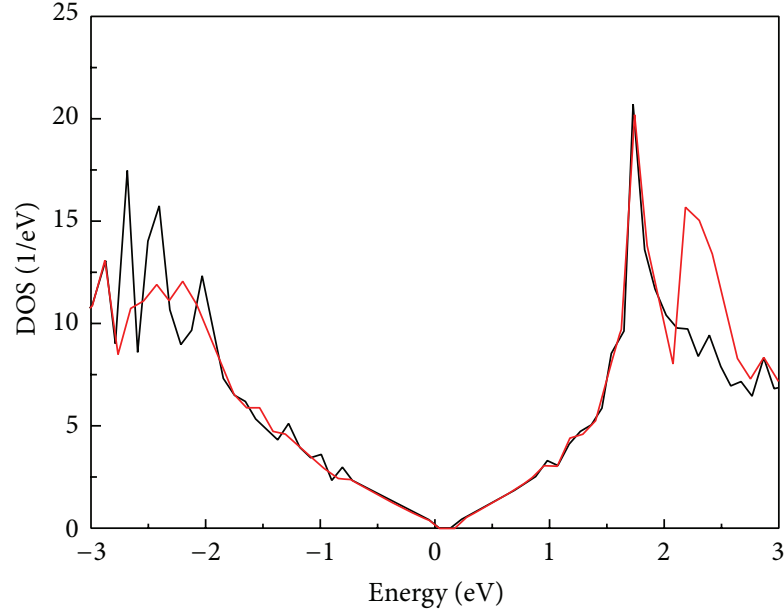

- G

- $\mathrm{CO}$ on $\mathrm{G}$

(a)

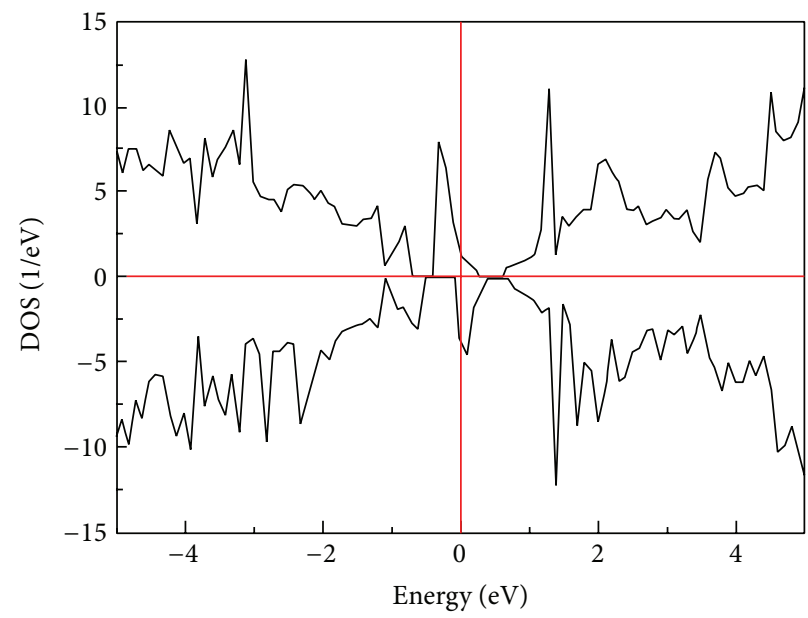

— DG

(c)
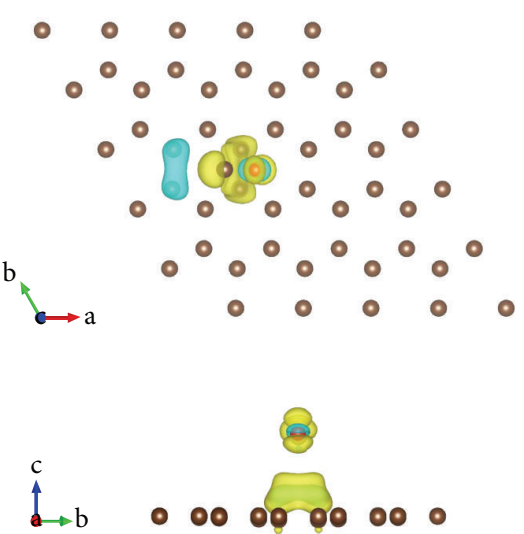

(e)

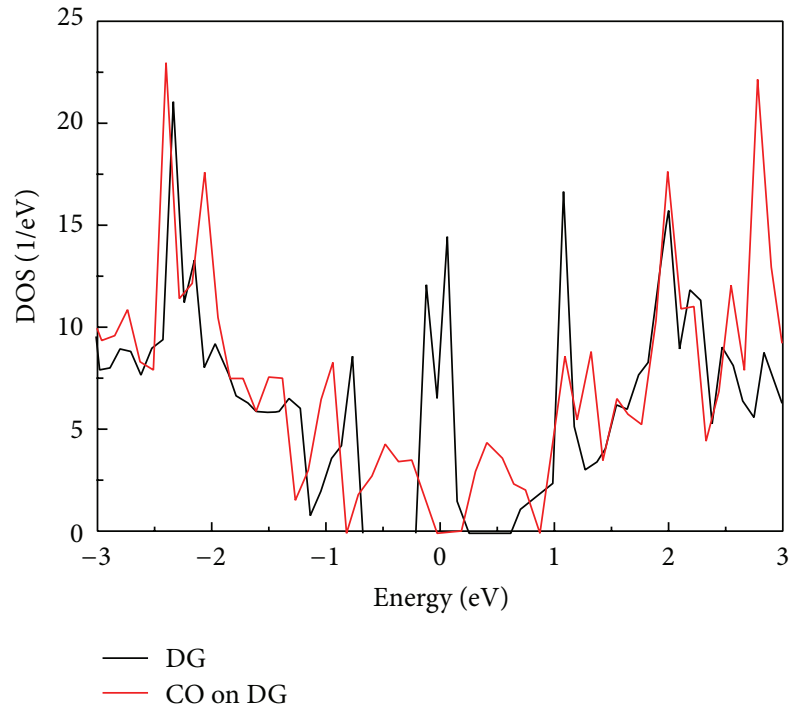

(b)

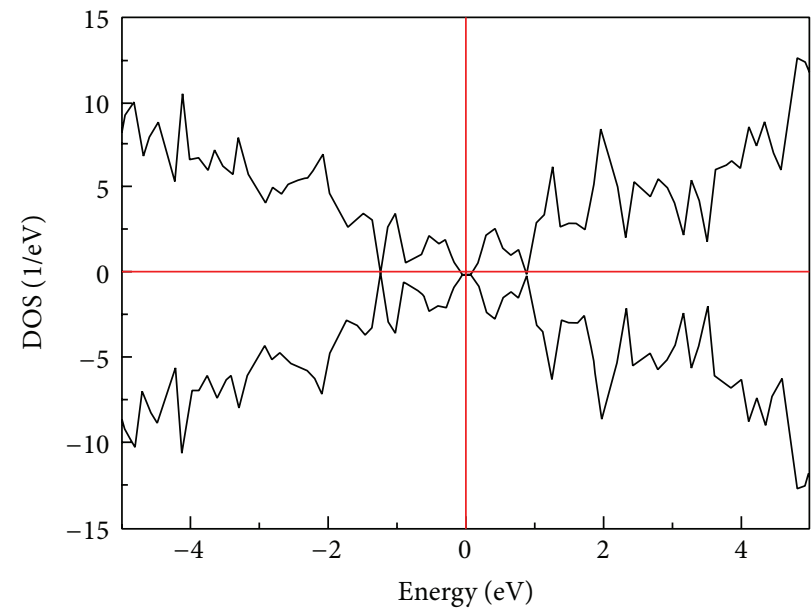

- $\mathrm{CO}$ on DG

(d)

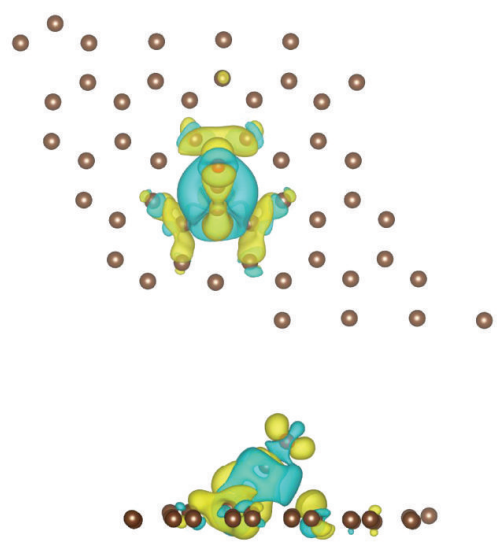

(f)

FIGURE 2: The DOS for (a) CO on pristine graphene and (b) CO on defective graphene. Spin-polarized density of states (DOS) for (c) defective graphene and (d) CO on defective graphene. The Femi level of the system is set to zero. Calculated differential charge densities for (e) CO on pristine graphene and (f) $\mathrm{CO}$ on defective graphene. Blue color and yellow color show the areas with $\Delta \rho<0$ and $\Delta \rho>0$, respectively. Note that different isosurface levels were used in plots (e) and (f): $0.0002 \mathrm{e} / \AA^{3}$ for the former and $0.002 \mathrm{e} / \AA^{3}$ for the latter. 


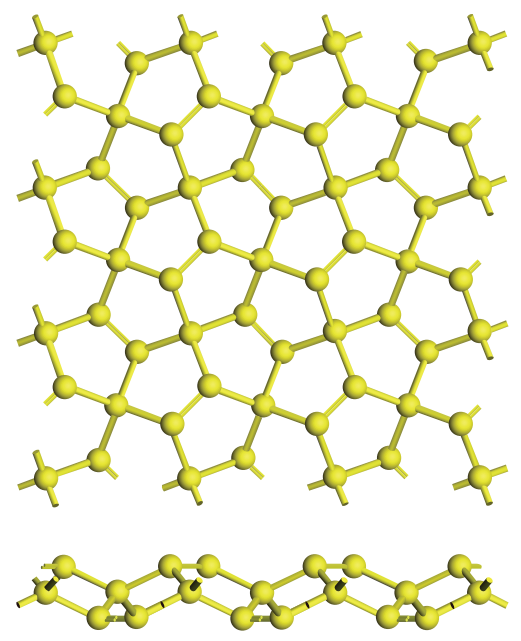

(a)
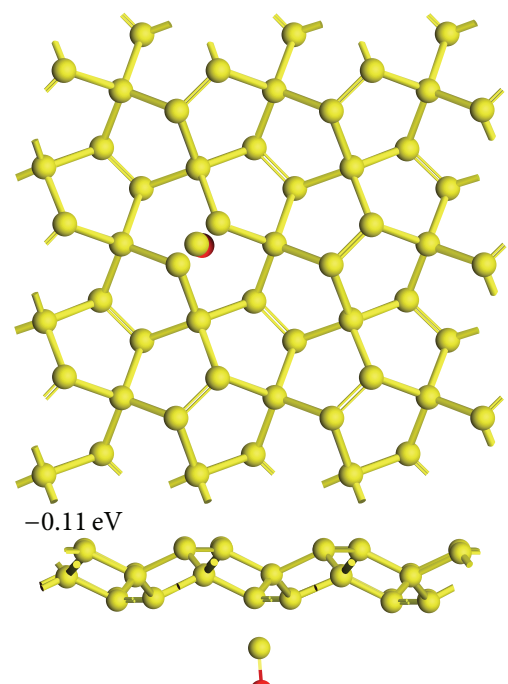

(c)

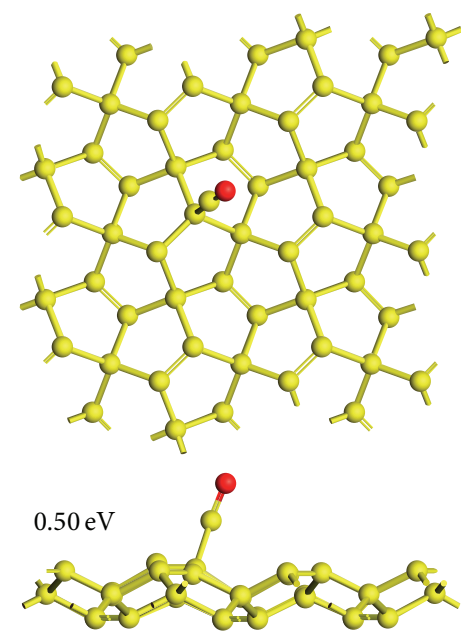

(b)

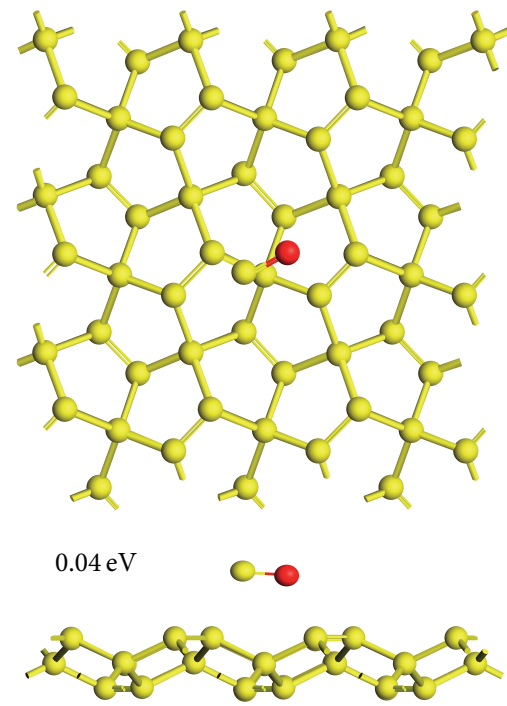

(d)

Figure 3: Top and side views of (a) the penta-graphene sheet, (b) to (d) CO adsorbed on the penta-graphene sheet at different sites. The adsorption energies are also shown in the figure.

former methods utilized the environmental dependent $\mathrm{vdW}$ parameters in DFT calculations. The vdW-DF functional gives the highest binding energy due to the well-known overly repulsive exchange part (revPBE) used in this approach [18]. Although the $E_{\text {ad }}$ ranges from -1.80 to $-2.25 \mathrm{eV}$, their related distances for all functionals are the same (1.33 $\AA$ ). This indicates that the adsorption distance is determined by the covalent bonding, rather than the $\mathrm{vdW}$ interactions, for the chemisorbed systems.

To better understand the change in the electronic structure caused by $\mathrm{CO}$ adsorption, we computed the electronic density of states (DOS) for CO on G and further compared it with that of CO on DG. As shown in Figure 2(a), almost no electron orbital overlaps between $\mathrm{CO}$ molecule and the graphene in the $\mathrm{CO}$ on $\mathrm{G}$ system due to the physisorptive nature of bonding in this case. On the contrary, the DOS plots of $\mathrm{CO}$ and the defective graphene in the CO on DG system are significantly hybridized (Figure 2(b)), clearly implying the strong chemisorption between the $\mathrm{CO}$ molecule and the defective graphene. It is inferred that the adsorption of CO on DG brings apparent perturbation to electronic properties of the defective graphene. Also, the spin-polarized DOS analysis shows that the DG is magnetic $(1.68 \mu \mathrm{B})$ whilst the magnetism vanishes upon the CO adsorption (see Figures 2(c) and 2(d)).

To obtain the charge redistribution after the adsorption process, we further computed the differential charge densities (DCD), which is defined as

$$
\Delta \rho=\rho_{\mathrm{CO} / \text { Graphene }}-\rho_{\text {Graphene }}-\rho_{\mathrm{CO}}
$$

where $\rho_{\mathrm{CO} / \text { Graphene }}$ represents the total electronic density for the $\mathrm{CO} /$ graphene system and $\rho_{\text {Graphene }}$ and $\rho_{\mathrm{CO}}$ are the densities for the isolated graphene (or defective graphene) and CO molecule, respectively. In Figures 2(e) and 2(f), we present the results of $\Delta \rho$ at the CO-G and CO-DG interfaces. 
Very weak charge transfer can be observed from graphene (blue color, $\Delta \rho<0$ ) to the CO molecule (yellow color, $\Delta \rho>$ 0 ) in Figure 2(e). For CO-DG in Figure 2(f), a significant charge transfer is observable from $\mathrm{CO}$ to defective graphene.

Finally, we made a comparison of graphene with the newly proposed penta-graphene [33] to confirm the better performance for the defective graphene as a $\mathrm{CO}$ gas sensor. As shown in Figure 3(a), the penta-graphene possesses $54 \mathrm{C}$ atoms, and the optimized lattice constants are $10.69 \AA$ from $\mathrm{PBE}+\mathrm{vdW}$ method. The total thickness of the relaxed structure is $1.26 \AA$ and the buckling is $0.63 \AA$. There are two kinds of $\mathrm{C}$ atoms, the $s p^{3}$ - and the $s p^{2}$-hybridized $\mathrm{C}$ atoms named $\mathrm{C} 1$ and $\mathrm{C} 2$, respectively. The $\mathrm{C} 1-\mathrm{C} 2$ is $1.53 \AA$ and $\mathrm{C} 2-\mathrm{C} 2$ is $1.33 \AA$, while the bond angle of $\mathrm{C} 2-$ $\mathrm{C} 1-\mathrm{C} 2$ is $131.1^{\circ}$. Three different configurations are shown in Figures 3(b) to 3(d). The most favorable configuration is site c with an $E_{\mathrm{ad}}$ of $-0.11 \mathrm{eV}$ and a distance of $2.19 \AA$. The remaining two configurations are both unstable due to the positive $E_{\text {ad }}$. Then we calculated the formation energy of the graphene $(-9.28 \mathrm{eV})$ and penta-graphene $(-8.39 \mathrm{eV})$ per atom, respectively. We found the formation energy of graphene is lower than that of penta-graphene.

\section{Conclusions}

In summary, the interactions between $\mathrm{CO}$ molecule and the pristine and defective graphene layers using different vdWinclusive functionals were investigated. $\mathrm{CO}$ is physisorbed on the perfect graphene sheets, along with low adsorption energy and almost no charge transfer. In contrast, the defective graphene is reactive to bond with $\mathrm{CO}$ molecule, and their interactions tune the electronic and magnetic properties of the adsorption system. Our calculations demonstrate the potential application of defective graphene as a highly sensitive $\mathrm{CO}$ gas sensor.

\section{Conflict of Interests}

The authors declare that there is no conflict of interests regarding the publication of this paper.

\section{Acknowledgments}

The authors gratefully acknowledge the financial support of National Natural Science Foundation of China (21403113, 51225102, and 2012CB932203), Program for New Century Excellent Talents in University from Chinese Ministry of Education, the Fundamental Research Funds for the Central Universities (30915011330), and a Project Funded by the Priority Academic Program Development of Jiangsu Higher Education Institutions.

\section{References}

[1] O. Leenaerts, B. Partoens, and F. M. Peeters, "Adsorption of $\mathrm{H}_{2} \mathrm{O}, \mathrm{NH}_{3}, \mathrm{CO}, \mathrm{NO}_{2}$, and $\mathrm{NO}$ on graphene: a first-principles study," Physical Review B, vol. 77, Article ID 125416, 2008.
[2] F. Schedin, A. K. Geim, S. V. Morozov et al., "Detection of individual gas molecules adsorbed on graphene," Nature Materials, vol. 6, no. 9, pp. 652-655, 2007.

[3] H. Choi, J. S. Choi, J.-S. Kim et al., "Flexible and transparent gas molecule sensor integrated with sensing and heating graphene layers," Small, vol. 10, no. 18, pp. 3685-3691, 2014.

[4] W. Hu, N. Xia, X. Wu, Z. Li, and J. Yang, "Silicene as a highly sensitive molecule sensor for $\mathrm{NH}_{3}, \mathrm{NO}$ and $\mathrm{NO}_{2}$," Physical Chemistry Chemical Physics, vol. 16, pp. 6957-6962, 2014.

[5] G. Mazzamuto, A. Tabani, S. Pazzagli et al., "Single-molecule study for a graphene-based nano-position sensor," New Journal of Physics, vol. 16, Article ID 113007, 2014.

[6] A. H. Reshak and S. Auluck, "Adsorbing $\mathrm{H}_{2} \mathrm{~S}$ onto a single graphene sheet: a possible gas sensor," Journal of Applied Physics, vol. 116, Article ID 103702, 2014.

[7] L. Shao, G. Chen, H. Ye et al., "Sulfur dioxide molecule sensors based on zigzag graphene nanoribbons with and without $\mathrm{Cr}$ dopant," Physics Letters A, vol. 378, no. 7-8, pp. 667-671, 2014.

[8] G. S. Kulkarni, K. Reddy, Z. Zhong, and X. Fan, "Graphene nanoelectronic heterodyne sensor for rapid and sensitive vapour detection," Nature Communications, vol. 5, article 4376, 2014.

[9] W. Yuan, A. Liu, L. Huang, C. Li, and G. Shi, "Highperformance $\mathrm{NO}_{2}$ sensors based on chemically modified graphene," Advanced Materials, vol. 25, no. 5, pp. 766-771, 2013.

[10] L. Bai and Z. Zhou, "Computational study of B- or N-doped single-walled carbon nanotubes as $\mathrm{NH}_{3}$ and $\mathrm{NO}_{2}$ sensors," Carbon, vol. 45, no. 10, pp. 2105-2110, 2007.

[11] C. Matranga, L. Chen, B. Bockrath, and J. K. Johnson, "Displacement of $\mathrm{CO}_{2}$ by Xe in single-walled carbon nanotube bundles," Physical Review B, vol. 70, no. 16, Article ID 165416, 7 pages, 2004.

[12] L. B. Da Silva, S. B. Fagan, and R. Mota, "Ab initio study of deformed carbon nanotube sensors for carbon monoxide molecules," Nano Letters, vol. 4, no. 1, pp. 65-67, 2004.

[13] J. A. Robinson, E. S. Snow, Ş. C. Badescu, T. L. Reinecke, and F. K. Perkins, "Role of defects in single-walled carbon nanotube chemical sensors," Nano Letters, vol. 6, no. 8, pp. 1747-1751, 2006.

[14] K. S. Novoselov, A. K. Geim, S. V. Morozov et al., "Electric field in atomically thin carbon films," Science, vol. 306, no. 5696, pp. 666-669, 2004.

[15] W. Liu, A. Savara, X. Ren et al., "Toward low-temperature dehydrogenation catalysis: isophorone adsorbed on $\operatorname{Pd}(111)$," The Journal of Physical Chemistry Letters, vol. 3, no. 5, pp. 582586, 2012.

[16] Y. Zhang, Y. Chen, K. Zhou et al., "Improving gas sensing properties of graphene by introducing dopants and defects: a first-principles study," Nanotechnology, vol. 20, no. 18, Article ID 185504, 2009.

[17] R. Lv, M. C. dos Santos, C. Antonelli et al., "Large-area Si-doped graphene: controllable synthesis and enhanced molecular sensing," Advanced Materials, vol. 26, no. 45, pp. 7593-7599, 2014.

[18] W. Liu, A. Tkatchenko, and M. Scheffler, "Modeling adsorption and reactions of organic molecules at metal surfaces," Accounts of Chemical Research, vol. 47, no. 11, pp. 3369-3377, 2014.

[19] W. Liu, V. G. Ruiz, G.-X. Zhang et al., "Structure and energetics of benzene adsorbed on transition-metal surfaces: densityfunctional theory with van der Waals interactions including collective substrate response," New Journal of Physics, vol. 15, Article ID 053046, 2013. 
[20] W. Liu, J. Carrasco, B. Santra, A. Michaelides, M. Scheffler, and A. Tkatchenko, "Benzene adsorbed on metals: concerted effect of covalency and van der Waals bonding," Physical Review BCondensed Matter and Materials Physics, vol. 86, no. 24, Article ID 245405, 2012.

[21] P. Hohenberg and W. Kohn, "Inhomogeneous electron gas," Physical Review B, vol. 136, no. 3, pp. B864-B871, 1964.

[22] W. Kohn and L. J. Sham, "Self-consistent equations including exchange and correlation effects," Physical Review A, vol. 140, Article ID A1133, 1965.

[23] G. Kresse and J. Furthmüller, "Efficiency of ab-initio total energy calculations for metals and semiconductors using a plane-wave basis set," Computational Materials Science, vol. 6, no. 1, pp. 15-50, 1996.

[24] P. E. Blöchl, "Projector augmented-wave method," Physical Review B, vol. 50, no. 24, pp. 17953-17979, 1994.

[25] J. P. Perdew, K. Burke, and M. Ernzerhof, "Generalized gradient approximation made simple," Physical Review Letters, vol. 77, no. 18 , article $3865,1996$.

[26] A. Tkatchenko and M. Scheffler, "Accurate molecular van der Waals interactions from ground-state electron density and freeatom reference data," Physical Review Letters, vol. 102, Article ID 073005, 2009.

[27] S. Grimme, "Semiempirical GGA-type density functional constructed with a long-range dispersion correction," Journal of Computational Chemistry, vol. 27, no. 15, pp. 1787-1799, 2006.

[28] S. Grimme, J. Antony, S. Ehrlich, and H. Krieg, "A consistent and accurate $a b$ initio parametrization of density functional dispersion correction (DFT-D) for the 94 elements $\mathrm{H}-\mathrm{Pu}$," The Journal of Chemical Physics, vol. 132, no. 15, Article ID 154104, 2010.

[29] J. Klimeš, D. R. Bowler, and A. Michaelides, "Chemical accuracy for the van der Waals density functional," Journal of Physics: Condensed Matter, vol. 22, no. 2, Article ID 022201, 2010.

[30] J. Klimeš, D. R. Bowler, and A. Michaelides, "Van der Waals density functionals applied to solids," Physical Review B, vol. 83, no. 19, Article ID 195131, 13 pages, 2011.

[31] D. R. Cooper, B. D’Anjou, N. Ghattamaneni et al., "Experimental review of graphene," ISRN Condensed Matter Physics, vol. 2012, Article ID 501686, 56 pages, 2012.

[32] Y. Luo, CRC Handbook of Chemistry and Physics, CRC Press, Boca Raton, Fla, USA, 2010.

[33] S. Zhang, J. Zhou, Q. Wang, X. Chen, Y. Kawazoe, and P. Jena, "Penta-graphene: a new carbon allotrope," Proceedings of the National Academy of Sciences, vol. 112, no. 8, pp. 2372-2377, 2015. 

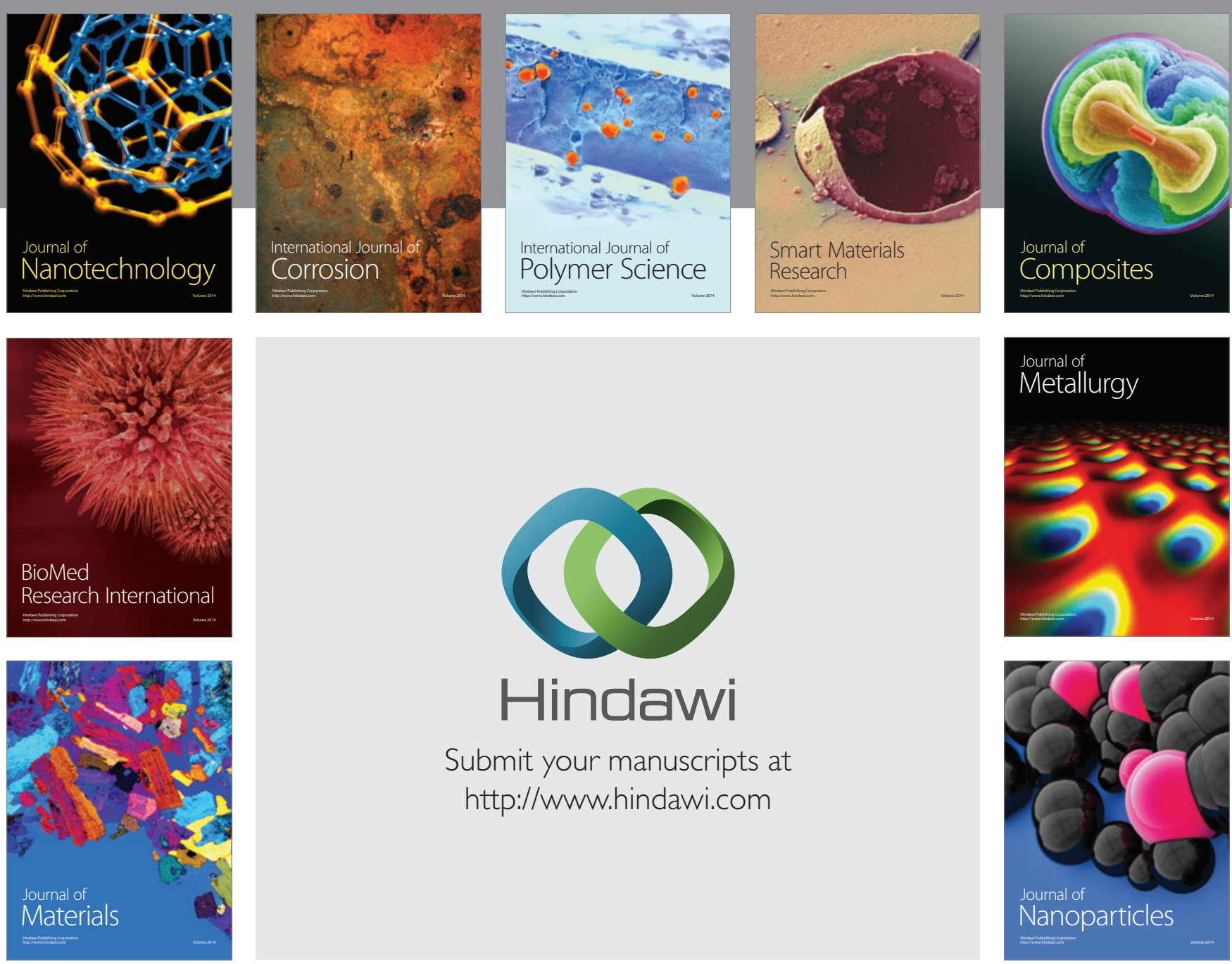

Submit your manuscripts at http://www.hindawi.com
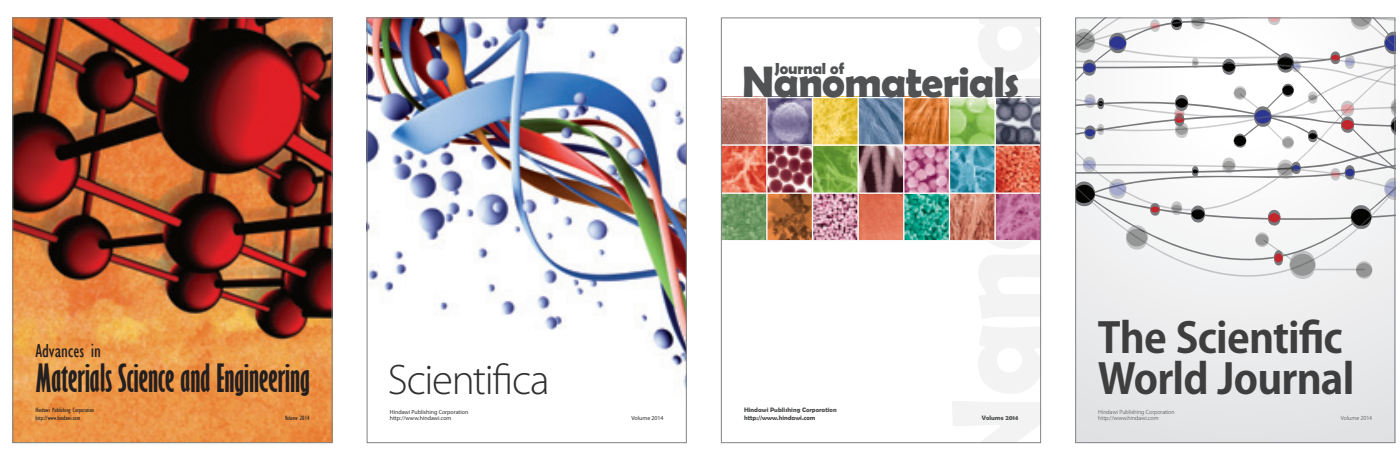

\section{The Scientific World Journal}
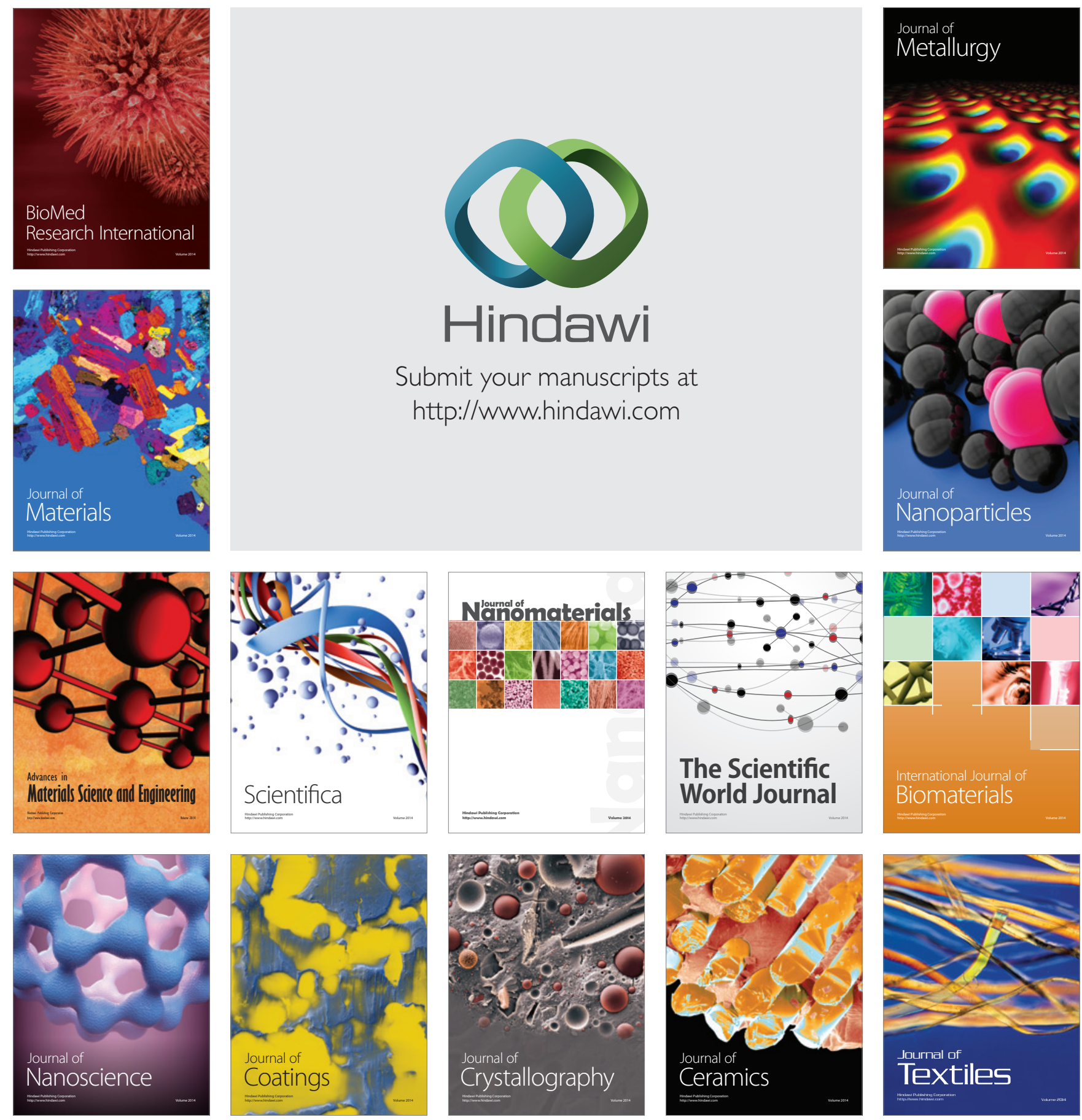